\title{
TheT cell plasma membrane lipid bilayer stages TCR-proximal signaling events
}

\section{Thomas Harder*}

35510 Butzbach, Germany

${ }^{*}$ Correspondence: tharder@faciendum.org

\section{INTRODUCTION}

The $\mathrm{T}$ cell plasma membrane lipid bilayer attracts the attention of immunologists as fundamental two-dimensional platform for molecular networks which mediate $\mathrm{T}$ cell antigen receptor (TCR) activation responses. Plasma membrane lipids critically influence formation and activity of signaling networks linked to the $\mathrm{T}$ cell plasma membrane. This opinion article outlines recent advances in understanding functional implications of their organization into highly dynamic raft lipid nanoassemblies which can be triggered to coalesce into ordered raft membrane phases and then serve as functional platforms for multimolecular TCR signaling machineries. Raft lipid dynamics are also incorporated into models of initiating TCR signal transduction. Hence, including the biochemical and biophysical complexity of the plasma membrane lipid bilayer is critical for systematic characterization of TCR-proximal signaling functions.

\section{FUNCTIONAL PLASMA MEMBRANE ORGANIZATION FOLLOWING T CELL ACTIVATION}

$\mathrm{T}$ cell activities are induced by TCR signals which are triggered by its interaction with a cognate peptide MHC (pMHC) ligand presented on the surface of an antigen-presenting cell (APC) or target cell. TCR-proximal signals are passed on from plasma membrane-associated signaling TCR microclusters to cytoplasmic and nuclear activities which mediate downstream immunological $\mathrm{T}$ cell effector functions.

A highly organized immunological synapse (IS) between the T cell and the cognate cell is marked by segregation of functionally distinctive supramolecular activation clusters (SMACs) into domains of the T cell plasma membrane (Huppa and Davis, 2003). Spatial organization of TCR activation in the contact region of the $\mathrm{T}$ cell with $\mathrm{T}$ cellactivating membrane surface was followed by video microscopy. These studies noted on-going TCR-proximal signaling in TCR microclusters which form in the periphery of the IS and are then transported centripetally. At the central SMAC TCR-proximal signaling is attenuated and TCR signaling complexes are tagged for endocytosis into the degradative lysosomal pathway (Campi et al., 2005; Varma et al., 2006).

Dynamic TCR signaling clusters in the plasma membrane were initially imaged by confocal microscopy of Jurkat $T$ cells plated on glass coverslips coated with $\alpha \mathrm{CD} 3$ TCRactivating antibody (Bunnell et al., 2002). In later studies, interactions of plasma membrane-anchored signaling marker proteins with antibody-induced TCR signaling clusters were assessed by tracking translational trajectories of single protein molecules and measuring their diffusional retardation at TCR signaling domains. These studies highlighted signaling-induced protein/ protein interactions as important drivers of TCR plasma membrane signaling clusters formation, while raft lipid-dependent interactions were not observed (Douglass and Vale, 2005). A related approach was used to determine diffusion coefficients of inner leaflet raft-anchored proteins relative to TCR signaling clusters. In these studies diffusion properties of membrane-linked marker proteins did indicate contributions of raft lipid- and actin-dependent interactions to diffusional retardation of cytoplasmic leaflet membrane raft-anchored proteins in the vicinity of TCR signaling clusters (Ike et al., 2003).

\section{NANOMETER-SCALE RAFT LIPID CONNECTIVITY AND COALESCENCE OF CONDENSED, ORDERED RAFT MEMBRANE PHASE AT TCR SIGNALING DOMAINS}

Insight into highly dynamic, nanometerscale sphingolipid/cholesterol raft assemblies in the plasma membrane of unstimulated cells was achieved by approaches which circumvent the diffraction-limit in resolving power of conventional lens-based light microscopy (Hell, 2009). Using these technologies it was possible to track dynamic nanoscale interactions of raft lipids in the plasma membrane (Eggeling et al., 2009). New strategies in correlation spectroscopy were used by He, Marguet and colleagues to show that phosphatidylinositol-3,4,5triphosphate $\left(\mathrm{PIP}_{3}\right)$-dependent Akt/PKB activation at the plasma membrane is controlled by nanoscale raft lipid-interactions in the cytoplasmic leaflet of the lipid bilayer (Lasserre et al., 2008).

Physical structure of TCR plasma membrane domains following receptor activation was monitored using fluorescent probes Laurdan and di-4-ANEPPDHQ which report on relative packing density of lipid membranes. These dyes revealed condensation of the plasma membrane at the IS which depended on TCR signaling and intact actin cytoskeleton (Gaus et al., 2005; Owen et al., 2010). Dense packing of these $\mathrm{T}$ cell plasma membrane sites indicated coalescence of ordered raft lipid domains at sites of TCR activation. In support of this notion, TCR signalingdependent membrane condensation was perturbed by incorporation of ordered membrane phase-disrupting lipid compounds; 7-ketocholesterol and polyunsaturated fatty acid (PUFA), into T cell membranes (Rentero et al., 2008; Zech et al., 2009). Coalescence and stabilization of ordered bilayer phases at TCR signaling plasma membrane domains is mediated by a vast molecular network of signaling proteins which was recently characterized by proteomic analyses (De Wet et al., 2011). Interestingly, disruption of plasma membrane condensation at TCR signaling sites correlated with reduced tyrosine phosphorylation in TCR signaling clusters and impeded assembly of TCR signaling protein complexes in the plasma membrane (Rentero et al., 2008), indicating 
contributions of both, ordered-phase lipid membrane and signaling protein networks, to the generation of TCR signaling clusters.

New mass spectrometric technology was developed which quantitatively charts molecular lipid composition of complex membranes. Mammalian cell membranes are made up of cholesterol, and thousands of glycerophospholipid- and sphingolipidspecies which differ in their polar headgroups, their fatty acid-positions, -lengths, and -saturation (Shevchenko and Simons, 2010). Charting the lipidome of isolated TCR signaling plasma membrane domains revealed that physical condensation of TCR signaling plasma membrane was indeed mirrored in its lipid chemistry. The TCR signaling lipidome accumulated molecular raft lipids; cholesterol, saturated phosphatidylcholine (PC) species, and sphingolipids (Zech et al., 2009). As interesting additional feature, the raft lipidome at TCR activation sites accumulates inner leaflet glycerophospholipid phosphatidylserine (PS) (Zech et al., 2009). A correlation between densely packed raft lipid domains and accumulation of phosphatylserine was observed in ordered raft lipid membranes of other systems (Lorizate et al., 2009; Fairn et al., 2011).

Influence of PS on the structure of TCR/CD3 receptor complex was studied by in vitro analyses. These studies showed that a peptide corresponding to the cytoplasmic portion of TCR $\zeta$-chain interacts with liposomes composed of negatively charged glycerophospholipids like PS and then adopts helical structure and is refractory to tyrosine phosphorylation (Aivazian and Stern, 2000). Similarly it was found that $\mathrm{CD} 3 \varepsilon$ cytoplasmic region specifically interacts with PS via basic amino acid residues in the $\mathrm{CD} 3 \varepsilon$ cytoplasmic protein segment $(\mathrm{Xu}$ et al., 2008). It was proposed that this interaction represents a safety switch to avoid erroneous TCR/CD3 tyrosine phosphorylation under resting $\mathrm{T}$ cell conditions (Kuhns and Davis, 2008). This proposal was subject to discussion following reports that tyrosine phosphorylation of CD3E cytoplasmic domain did not increase when these basic residues were mutated (Fernandes et al., 2010; Gagnon et al., 2010).

A role of PS in generation of signaling protein membrane networks at TCR triggering sites was suggested by reconstructing LATanchored TCR lipid/signaling protein network in vitro: tyrosine phosphorylated LAT was recombinantly expressed as membraneanchored variant in insect cells and inserted into liposomes. Nucleation of cooperative multimolecular signaling protein networks around these phosphotyrosineLAT-proteoliposomes effectively occurred on membranes which were composed of PS- but not of PC-lipid. This indicated the fundamental importance of the lipid bilayer platform and its composition for the nucleation of LAT-anchored TCR downstream signaling complexes (Sangani et al., 2009).

\section{IMPLICATION IN TCR TRIGGERING MECHANISMS}

In vitro reconstitution is indeed a powerful approach to reduce the complexity of collective systems to isolated features. This has also been performed with great success in model lipid membranes which feature cholesterol-dependent segregation of liquid ordered $\left(l_{o}\right)$ and liquid disordered $\left(l_{d}\right)$ phases (Ahmed et al., 1997). Immiscibility of artificial $l_{o}$ - and $l_{d}$-phases in model membranes recapitulates (but cannot be equated with Kaiser et al., 2009) segregation of ordered raft- and disordered non-raft phases in cell plasma membranes. It was shown that in isolated cell plasma membrane vesicles at $37^{\circ} \mathrm{C}$ connectivity of raft lipids does not suffice to segregate bulk lipid membrane phases. However, segregation of ordered and disordered phases in cell plasma membrane vesicles could be induced by choleratoxin $B$ subunit (CTB)-mediated crosslinking of raft GM1 ganglioside lipid component (Lingwood et al., 2008). These results suggest that resting cell plasma membranes are held below a critical threshold above which raft lipid connectivity causes bulk phase separation at physiological conditions. Segregation of ordered and disordered phases can then be triggered by passing this threshold with relatively small shifts in membrane status, for example by pentavalent crosslinking of GM1 using CTB. Hence, it can be envisioned that ligand-induced alterations of TCR configuration in the plasma membrane bilayer locally induces ordering of its lipid environment. This causes coupling of ligandengaged TCR to membrane-anchored Lck Src-family kinase which prefers an ordered lipid bilayer environment (Janes et al., 1999; Ike et al., 2003). Increased accessibility of Lck kinase to ligand-engaged TCR complex would then cause ITAM phosphorylation and receptor activation. Indeed, it was recently shown that a significant fraction of Lck kinase in $\mathrm{T}$ cells is in its active form prior to TCR activation and will phosphorylate its ITAM substrate in the TCR/CD3 complex once it gains access (Nika et al., 2010).

\section{OUTLOOK}

Induction of ordered raft phase by ligand engagement has been put forward as central element of models describing initial TCR triggering (van der Merwe and Dushek, 2011). Testing this experimentally poses the challenge of comparing structure of TCR lipid bilayer environment prior to and post ligand engagement and monitoring how ligand engagement causes raft phase coalescence in the immediate, possibly nanometer range TCR environment. This needs to be experimentally discriminated from segregation of micrometer scale ordered raft phase shown to be a consequence of TCR signaling at $\mathrm{T}$ cell activation domains.

\section{REFERENCES}

Ahmed, S. N., Brown, D. A., and London, E. (1997). On the origin of sphingolipid/cholesterol-rich detergent-insoluble cell membranes: physiological concentrations of cholesterol and sphingolipid induce formation of a detergent-insoluble, liquid-ordered lipid phase in model membranes. Biochemistry 36, 10944-10953.

Aivazian, D., and Stern, L. J. (2000). Phosphorylation of $\mathrm{T}$ cell receptor zeta is regulated by a lipid dependent folding transition. Nat. Struct. Biol. 7, 1023-1026.

Bunnell, S. C., Hong, D. I., Kardon, J. R., Yamazaki, T., Mcglade, C. J., Barr, V.A., and Samelson, L. E. (2002). $\mathrm{T}$ cell receptor ligation induces the formation of dynamically regulated signaling assemblies. J. Cell Biol. 158, 1263-1275.

Campi, G., Varma, R., and Dustin, M. L. (2005). Actin and agonist MHC-peptide complex-dependent $\mathrm{T}$ cell receptor microclusters as scaffolds for signaling. J. Exp. Med. 202, 1031-1036.

De Wet, B., Zech, T., Salek, M., Acuto, O., and Harder, T. (2011). Proteomic characterization of plasma membrane-proximal $\mathrm{T}$ cell activation responses. $J$. Biol. Chem. 286, 4072-4080.

Douglass, A. D., and Vale, R. D. (2005). Single-molecule microscopy reveals plasma membrane microdomains created by protein-protein networks that exclude or trap signaling molecules in T cells. Cell 121, 937-950.

Eggeling, C., Ringemann, C., Medda, R., Schwarzmann, G., Sandhoff, K., Polyakova, S., Belov, V. N., Hein, B., Von Middendorff, C., Schonle, A., and Hell, S. W. (2009). Direct observation of the nanoscale dynamics of membrane lipids in a living cell. Nature 457 , 1159-1162.

Fairn, G. D., Schieber, N. L., Ariotti, N., Murphy, S., Kuerschner, L., Webb, R. I., Grinstein, S., and Parton, R. G. (2011). High-resolution mapping reveals topologically distinct cellular pools of phosphatidylserine. J. Cell Biol. 194, 257-275. 
Fernandes, R. A., Yu, C., Carmo, A. M., Evans, E. J., Van Der Merwe, P.A., and Davis, S. J. (2010). What controls T cell receptor phosphorylation? Cell 142, 668-669.

Gagnon, E., Xu, C., Yang, W., Chu, H. H., Call, M.E., Chou, J. J., and Wucherpfennig, K. W. (2010). Response multilayered control of $\mathrm{T}$ cell receptor phosphorylation. Cell 142, 669-671.

Gaus, K., Chklovskaia, E., Fazekas De St Groth, B., Jessup, W., and Harder, T. (2005). Condensation of the plasma membrane at the site of $\mathrm{T}$ lymphocyte activation. $J$. Cell Biol. 171, 121-131.

Hell, S. W. (2009). Microscopy and its focal switch. Nat. Methods 6, 24-32.

Huppa, J. B., and Davis, M. M. (2003). T-cell-antigen recognition and the immunological synapse. Nat. Rev. Immunol. 3, 973-983.

Ike, H., Kosugi, A., Kato, A., Iino, R., Hirano, H., Fujiwara, T., Ritchie, K., and Kusumi, A. (2003). Mechanism of Lck recruitment to the T-cell receptor cluster as studied by single-molecule-fluorescence video imaging. Chemphyschem 4, 620-626.

Janes, P.W., Ley, S. C., and Magee, A. I. (1999). Aggregation of lipid rafts accompanies signaling via the T cell antigen receptor. J. Cell Biol. 147, 447-461.

Kaiser, H. J., Lingwood, D., Levental, I., Sampaio, J. L., Kalvodova, L., Rajendran, L., and Simons, K. (2009). Order of lipid phases in model and plasma membranes. Proc. Natl. Acad. Sci. U.S.A. 106, 16645-16650.

Kuhns, M. S., and Davis, M. M. (2008). The safety on the TCR trigger. Cell 135, 594-596.

Lasserre, R., Guo, X. J., Conchonaud, F., Hamon, Y., Hawchar, O., Bernard, A. M., Soudja, S. M., Lenne, P. F., Rigneault, H., Olive, D., Bismuth, G., Nunes, J. A., Payrastre, B., Marguet, D., and He, H. T. (2008).
Raft nanodomains contribute to Akt/PKB plasma membrane recruitment and activation. Nat. Chem. Biol. 4, 538-547.

Lingwood, D., Ries, J., Schwille, P., and Simons, K. (2008). Plasma membranes are poised for activation of raft phase coalescence at physiological temperature. Proc. Natl. Acad. Sci. U.S.A. 105, 10005-10010.

Lorizate, M., Brugger, B., Akiyama, H., Glass, B., Muller, B., Anderluh, G., Wieland, F. T., and Krausslich, H. G. (2009). Probing HIV-1 membrane liquid order by Laurdan staining reveals producer cell-dependent differences. J. Biol. Chem. 284, 22238-22247.

Nika, K., Soldani, C., Salek, M., Paster, W., Gray, A., Etzensperger, R., Fugger, L., Polzella, P., Cerundolo, V., Dushek, O., Hofer, T., Viola, A., and Acuto, O. (2010). Constitutively active Lck kinase in T cells drives antigen receptor signal transduction. Immunity 32, 766-777.

Owen, D. M., Oddos, S., Kumar, S., Davis, D. M., Neil, M. A., French, P. M., Dustin, M. L., Magee, A. I., and Cebecauer, M. (2010). High plasma membrane lipid order imaged at the immunological synapse periphery in live T cells. Mol. Membr. Biol. 27, 178-189.

Rentero, C., Zech, T., Quinn, C. M., Engelhardt, K., Williamson, D., Grewal, T., Jessup, W., Harder, T., and Gaus, K. (2008). Functional implications of plasma membrane condensation for $\mathrm{T}$ cell activation. PLoS ONE 3, e2262.

Sangani, D., Venien-Bryan, C., and Harder, T. (2009). Phosphotyrosine-dependent in vitro reconstitution of recombinant LAT-nucleated multiprotein signalling complexes on liposomes. Mol. Membr. Biol. 26, 159-170.
Shevchenko, A., and Simons, K. (2010). Lipidomics: coming to grips with lipid diversity. Nat. Rev. Mol. Cell Biol. 11, 593-598.

Van Der Merwe, P.A., and Dushek, O. (2011).Mechanisms for T cell receptor triggering. Nat. Rev. Immunol. 11, 47-55.

Varma, R., Campi, G., Yokosuka, T., Saito, T., and Dustin, M.L. (2006). T cell receptor-proximal signals are sustained in peripheral microclusters and terminated in the central supramolecular activation cluster. Immunity 25, 117-127.

Xu, C., Gagnon, E., Call, M. E., Schnell, J. R., Schwieters, C. D., Carman, C. V., Chou, J. J., and Wucherpfennig, K. W. (2008). Regulation of T cell receptor activation by dynamic membrane binding of the CD3epsilon cytoplasmic tyrosine-based motif. Cell 135, 702-713.

Zech, T., Ejsing, C. S., Gaus, K., De Wet, B., Shevchenko, A., Simons, K., and Harder, T. (2009). Accumulation of raft lipids in T-cell plasma membrane domains engaged in TCR signalling. EMBO J. 28, 466-476.

Received: 28 November 2011; accepted: 29 February 2012; published online: 20 March 2012.

Citation: Harder T (2012) The T cell plasma membrane lipid bilayer stages TCR-proximal signaling events. Front. Immun. 3:50. doi: 10.3389/fimmu.2012.00050 This article was submitted to Frontiers in T Cell Biology, a specialty of Frontiers in Immunology.

Copyright (c) 2012 Harder. This is an open-access article distributed under the terms of the Creative Commons Attribution Non Commercial License, which permits non-commercial use, distribution, and reproduction in other forums, provided the original authors and source are credited. 